American Journal of Biochemistry and Biotechnology 4 (3): 304-316, 2008

ISSN 1553-3468

(C) 2008 Science Publications

\title{
NifH: Structural and Mechanistic Similarities with Proteins Involved in Diverse Biological Processes
}

\author{
Surobhi Lahiri, Lakshmi Pulakat and Nara Gavini \\ Department of Biological Sciences, \\ Mississippi State University, Mississippi State, MS 39762, USA
}

\begin{abstract}
The NifH protein is a subunit of the nitrogenase enzyme that catalyzes the reduction of atmospheric nitrogen to ammonia. This protein contains highly conserved regions including the nucleotide binding sites, metal center ligands and the Switch I and Switch II domains. A number of proteins have structural and mechanistic similarities as well as evolutionary relationships with the NifH protein, notable among them being: light independent protochlorophyllide (Pchlide) reductase (ChlL/FrxC or bChL), arsenite pump ATPase (ArsA), 2-hydroxyglutaryl dehydratase Component A (CompA) involved in glutamate degradation and MinD that functions in spatial regulation of cell division. Although involved in very diverse biological processes, these proteins share an underlying common structural framework. This review mainly focuses on the structural similarities of these proteins with the NifH protein and discusses recent reports of complementation studies involving NifH and few of the proteins mentioned.
\end{abstract}

Key words: $\mathrm{NifH}, \mathrm{ChlL}, \mathrm{ArsA}, \mathrm{CompA}$, MinD, domain conservation, complementation

\section{INTRODUCTION}

The nitrogenase enzyme is a two-component system that consists of the iron protein (Fe-protein or $\mathrm{NifH}$ ) and molybdenum-iron protein (MoFe-protein) working in concert to effect nitrogen reduction ${ }^{[1-4]}$. The $64 \mathrm{kDa}$ homodimeric NifH protein has two ATP binding domains and one $[4 \mathrm{Fe}-4 \mathrm{~S}]$ cluster per homodimer. It supplies energy by ATP hydrolysis and transfers electrons from reduced ferredoxin or flavodoxin to the MoFe-protein ${ }^{[3]}$. It can bind up to two $\mathrm{MgADP}$ and/or MgATP molecules per dimer and is essential for coupling nucleotide hydrolysis to electron transfer for eventual nitrogen reduction ${ }^{[5,6]}$. It also participates in the biosynthesis and insertion of the FeMo-cofactor into the MoFe protein ${ }^{[7-10]}$. In the past, various proteins have been revealed to have structural and mechanistic similarities as well as evolutionary relationships with the NifH protein, notable among them being: light independent protochlorophyllide (Pchlide) reductase (ChlL/FrxC or bChL), arsenite pump ATPase (ArsA), 2-hydroxyglutaryl dehydratase Component A (CompA) involved in glutamate degradation and MinD that functions in spatial regulation of cell division ${ }^{[11-16]}$. Although involved in diverse biological processes, these proteins have been found to bear considerable structural resemblance to the
NifH protein. Mechanistically, they do play a role similar to NifH in their respective complexes, based upon the presence of ATP binding motifs in each of them ${ }^{[17-21]}$. Whereas ChlL, ArsA and MinD belong to the same superfamily of proteins as NifH, known as the P-loop containing nucleoside triphosphate hydrolases, the Component $\mathrm{A}$ of 2-hydroxyglutaryl dehydratase (CompA) is a member of the Actin-like ATPase domain superfamily, as designated by the SCOP database ${ }^{[22]}$. An analogy between NifH and ChlL, ArsA, CompA and MinD lies in the organization of these proteins as members of their respective two component systems. In the nitrogenase complex, NifH functions as the obligate electron donor to its specific partner, the MoFe protein. This intermolecular electron transfer process requires ATP hydrolysis. In a similar manner, for the dehydratase system, CompA is the site of ATP hydrolysis and is the electron donor to the sec component, CompD ${ }^{[19]}$, ArsA exists in a complex with the ArsB protein forming an arsenite-antimonite $[\mathrm{As}(\mathrm{III}) / \mathrm{Sb}(\mathrm{III})]-$ translocating ATPase. As a result of the ATP hydrolysis in ArsA, a more compact conformation of the enzyme is generated, allowing the vectorial movement of the arsenite or antimonite ions into $\mathrm{ArsB}^{[13]}$; It has been speculated that the MinDMinE membrane complex is analogous to the NifHMoFe complex and results in ATP hydrolysis, as a

Corresponding Author: Nara Gavini, Department of Biological Sciences, Mississippi State University, Mississippi State, MS 39762, USA 


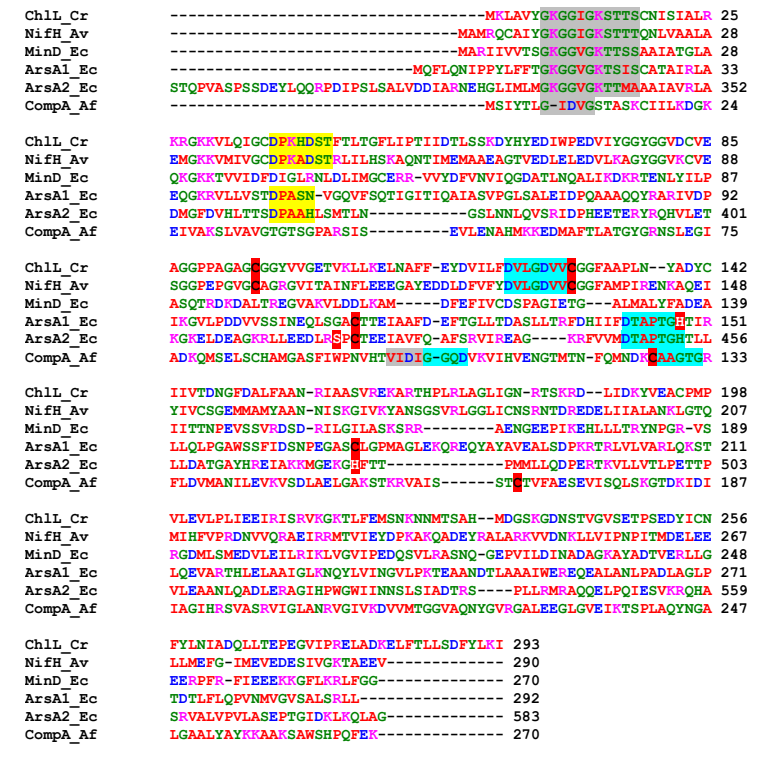

Fig. 1: Protein sequence alignment of NifH, ChlL, ArsA1, ArsA2, MinD and CompA. The residues are colored as red, blue, magenta, green and gray based on their hydrophobicity and other properties. Regions highlighted are: the nucleotide binding domains in gray; the Switch I in yellow; the Switch II in cyan. Cysteine residues highlighted in red are the ligands for the metal centers of their respective proteins. The histidine residue highlighted in red is one of the ligands for the $\mathrm{Sb}$ (III) center of ArsA. It should be noted that ArsA2 sequence includes the 'linker' stretch of residues of the ArsA protein (Cr: Chlamydomonas reinhardtii, Av: Azotobacter vinelandii, Ec: Escherichia coli, Af: Acidaminococcus fermentas)

consequence of which, MinD and MinE are released from the membrane ${ }^{[11]}$; Comparison of the molecular architecture between nitrogenase and the lightindependent Pchlide reductase has shown that the NifH counterpart in the light independent Pchlide reductase is ChlL. ChlL is suggested to be involved in ATPdependent transfer of electrons from a reductant, such as ferredoxin, to the ChlB-ChlN complex via the Fe:S center $^{[14]}$. Thus, in a manner similar to $\mathrm{NifH}$, the proteins ArsA, ChlL, CompA and MinD also couple hydrolysis of nucleoside triphosphates to redox reactions from a metallic cluster.

The protein sequence comparison of NifH, ArsA, ChlL, CompA and MinD gives an overview of their similar regions (Fig. 1). However, it is difficult to detect protein relationships and the presence of different

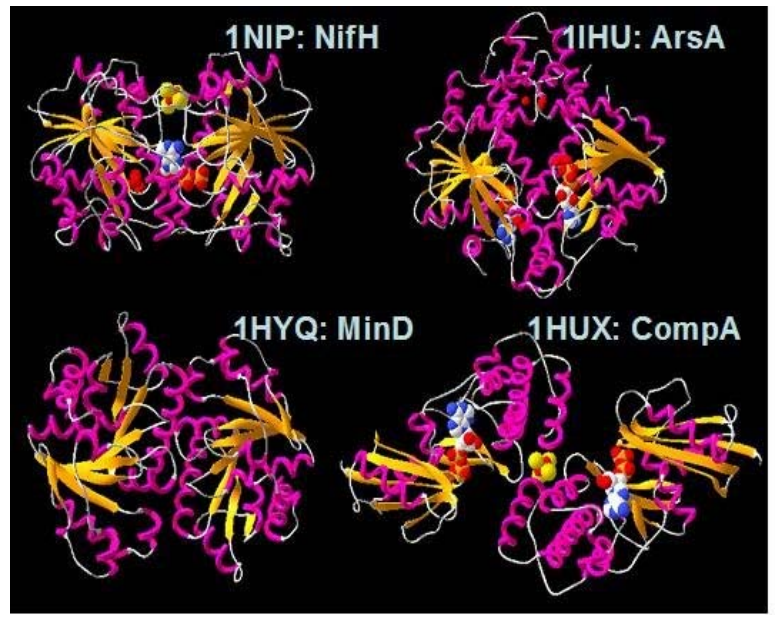

Fig. 2: 3-D representation of NifH, ArsA, MinD and CompA. The structures are colored on the basis of secondary structures. The $\alpha$-helices are shown in magenta, $\beta$-strands in orange and coils/loops are in gray. The yellow spacefilled structure in $\mathrm{NifH}$ and CompA represent the [4Fe-4S] cluster. The red spacefilled structures in ArsA show the $\mathrm{Sb}(\mathrm{III}) / \mathrm{As}$ (III) metal center. CPK colored spacefilled structures in NifH, ArsA and CompA indicate the bound MgADP

domains by direct comparisons of sequences belonging to different protein families and it is more reasonable to determine the presence or absence of domains and their family relationships by comparison of their threedimensional structures (Fig. 2). How proteins with similar protein folding patterns perform varied functions is a prevalent question and it has been suggested that popular folding patterns have thermodynamic advantages enabling them to be stabilized by random sequences and these few advantageous folds can probably tolerate various primary structures and therefore perform different functions ${ }^{[23]}$. The structural comparison of each of the four proteins with $\mathrm{NifH}$ creates a general model that indicates their common ancestry at some point. The functions of NifH, ArsA and ChlL, which are broadly nitrogen fixation, arsenite resistance and photosynthesis respectively, further point out to the very first evolutionarily important processes on earth. It has been speculated that the global functions of the $\mathrm{NifH}$ homologs may have varied with either change in nucleotide specificity, the incorporation of new functions or the loss of others ${ }^{[24]}$. A thorough analysis of the comparative structural details of these proteins allows a better understanding of their relationship to each other. Consequently, derived from the comparison 
of NifH with ChlL, ArsA, CompA and MinD, greater insight into their structure based function and phylogeny can be obtained.

\section{NifH: THE STRUCTURAL TEMPLATE}

The $2.9 \AA$ crystal structure of the NifH protein from Azotobacter vinelandii was obtained by Georgiadis et al. in $1992^{[5]}$. It revealed that each of the subunits of the NifH dimer consisted of mixed $\alpha$ helix/ $\beta$-sheet polypeptide fold, with a consensus topology of an eight-stranded $\beta$-sheet flanked by nine $\alpha$-helices. A $\beta$-sheet core in each monomer was formed of one short antiparallel and seven parallel $\beta$-strands. The binding sites critical to NifH function were determined by loops at the carboxy-terminal ends of several $\beta$-strands. The Nucleotide Binding Sites (NBSs) were situated in the loops following $\beta 1$ and $\beta 2$ and the cysteine ligands (C 97 and 132) for the [4Fe-4S] cluster were in the loops following $\beta 5$ and $\beta 6^{[5,25]}$. Both cluster ligands are located near the amino terminal ends of $\alpha$ helices that are directed toward the cluster. As indicated by spectroscopic studies, a prominent feature of the $\mathrm{Fe}$ protein is the exposure of the $[4 \mathrm{Fe}-4 \mathrm{~S}]$ cluster to the solvent ${ }^{[26]}$, which is also probably one of the reasons that it is an oxygen sensitive protein. In addition to the [4Fe-4S] cluster, there are numerous Van der Waals and polar interactions in the interface beneath the cluster that help stabilize the dimer structure.

$\mathrm{NifH}$ displays structural similarity to other nucleotide-binding proteins including signal transduction molecules such as G-proteins and ras and energy transduction systems such as myosin ${ }^{[27]}$. It is known to be similar to the nucleotide binding proteins based on the presence of the following structural features ${ }^{[28,29,30]}$ : (i) mainly parallel $\beta$-sheets flanked by $\alpha$-helices (ii) a phosphate-binding loop (P-loop) or Walker A motif ${ }^{[31]}$, containing the G-X-X-X-X-G-K$\mathrm{S} / \mathrm{T}$ consensus sequence and (iii) two switch regions, Switch I and Switch II that interact with the $\gamma$ phosphate group of the bound nucleoside triphosphate. Analysis of the position of a bound ADP molecule in the X-ray structure of the Fe protein from Azotobacter vinelandii ${ }^{[5]}$ and the properties of other site-specifically altered Fe proteins ${ }^{[8,27,32-35]}$, has established the position of the nucleotide binding sites, one on each subunit, $19 \AA$ away from the [4Fe-4S] cluster. The nucleotidedependent switch regions are responsible for communication between the sites responsible for nucleotide binding and hydrolysis and the [4Fe-4S] cluster of the Fe protein and the docking interface that interacts with the MoFe protein upon macromolecular complex formation. In A. vinelandii $\mathrm{NifH}$, residues 38 to 43 and 125 to 132 form the Switch I and Switch II respectively. The switch regions undergo conformational changes upon hydrolysis to the nucleoside diphosphate with consequent loss of the interaction with the terminal phosphate group. Substitutions in the nucleotide dependent switch regions of the NifH of Azotobacter vinelandii showed that the altered NifH proteins formed a trapped complex subsequent to a single electron transfer event ${ }^{[36]}$. The studies suggested that whereas in the structure of the native enzyme the analogous interaction between the side chains of D39 and 125 was precluded due to electrostatic repulsion, the D39N substitution allowed the formation of a hydrogen bond between the Switch I D39 and the Switch II D125; this demonstrated that the electrostatic repulsion between D39 and D125 was important for dissociation of the $\mathrm{Fe}$ protein: $\mathrm{MoFe}$ protein complex during catalysis ${ }^{[36]}$. Thus, the switch regions play a critical role in transmitting information concerning the nucleotide state to other effector molecules that bind to these regions.

Investigations of the dimer interface of the $\mathrm{NifH}$ protein have shown that in the NifH dimer, residues with $>30 \AA$ of buried surface area include $\mathrm{K} 41$, E 92 , P 93, V 95, A 98, D 129, V 130, V 131, C 132, M 156, Y 159, K 166 and K 170. These residues, along with other residues adjacent in the sequence, mediate the subunit-subunit interactions through a series of primarily polar (hydrogen bond and salt-bridge) interactions ${ }^{[24]}$. The $\mathrm{C}$-terminus of $\mathrm{NifH}$ has been speculated to provide an additional degree of intersubunit interaction in $\mathrm{NifH}$ as residues from this region wrap around the body of the opposing subunit and enhance the overall stabilization of the NifH dimer. Also, in the C-terminus region, cross-subunit saltbridges have been known to form between K 224-E 277 and $\mathrm{K} 233-\mathrm{E} 287^{[24]}$.

An integral part of the nitrogenase mechanism is MgATP binding to the Fe protein and hydrolysis by the Fe protein-MoFe protein complex. Through the studies of Jang et al., it was confirmed that the protein interactions with the $\mathrm{Mg} 2+$ were essential for the transduction of the nucleotide hydrolysis event ${ }^{[37]}$. Analysis of the MgADP bound crystallized Fe protein revealed that $\mathrm{S} 16$ binds the $\mathrm{Mg}^{2+}$ and $\alpha, \beta$ and $\delta$ phosphates of nucleotides and appears to form a hydrogen bond with D125 of the switch II region ${ }^{[27,37]}$. Yet another region found to be critical for the MgATPinduced conformational change was the highly conserved span of the Fe protein around A157 located at the subunit interface. This region is part of the $\alpha 5$ 
helix extending from residue 151 to 176 at the subunit interface and shows substantial difference when the free Fe protein structure is compared with the structure of the $\mathrm{Fe}$ protein in the MgATP bound complex. Moreover, the mutation of A 157 to Ser resulted in a protein that could still bind MgATP normally but was unable to undergo the MgATP-induced conformational change $^{[8]}$. Further insight into MgATP binding and hydrolysis was obtained by studies based on residues K 15 and D 125. It was suggested that the breaking of a salt bridge between these two residues by MgATP binding was responsible for triggering a conformational change $\mathrm{e}^{[33,34]}$.

The substrate reduction mechanism of the nitrogenase enzyme involves the key step of MoFe protein docking on the $\mathrm{Fe}$ protein. Recent studies dealing with the biochemical and structural characterization of the crosslinked complex of nitrogenase have shown the specificity of the residues involved in the transient complex formation between the $\mathrm{Fe}$ and $\mathrm{MoFe}$ proteins. It was found that only E112 from one of the two NifH subunits and K400 of the NifK subunit were crosslinked, although these residues were surrounded by numerous other charged residues that could potentially participate in this process ${ }^{[17]}$. Most recently, when the $\mathrm{Fe}$ and $\mathrm{MoFe}$ proteins were cocrystallized under the conditions of (i) no nucleotide (ii) MgADP and (iii) MgAMPPCP (an MgATP analog), the Fe protein molecules were found to be capable of occupying different interaction sites on the MoFe protein $^{[38]}$; three separate docking areas were thereby identified on the surface of the MoFe protein ${ }^{[38]}$. Other studies that substituted Ala in place of F 125 of the $\alpha$ and $\beta$ subunits of the MoFe protein, separately and in combination, showed that the doubly substituted MoFe protein was unable to form a tight complex with the MgADP- $\mathrm{AlF}_{4}{ }^{-}$treated NifH or when using the altered L127 $\Delta \mathrm{NifH}$, thereby suggesting that the $\mathrm{F} 125$ residues were involved in an early event(s) that occurred upon component protein docking and could be involved in eliciting MgATP hydrolysis ${ }^{[39]}$.

Amongst the proteins that share a high level of structural-functional similarity and sequence identity/similarity with NifH Table 1 and 2, ChlL bears maximum closeness to this protein. However, the ChlL crystallographic structure is not yet available and several predictions of its structural features have been made considering its likeness to $\mathrm{NifH}^{[14]}$, Table 1.

\section{ChIL: LIGHT-INDEPENDENT CHLOROPHYLL BIOSYNTHESIS}

The reduction of the C17-C18 double bond of the D-ring of protochlorophyllide (PChlide) to chlorophyllide (Chlide) is a major step in the chlorophyll biosynthesis pathway. The two pathways for this process involve catalysis by the enzymes NADPH : PChlide oxidoreductase (POR) and the lightindependent PChlide reductase (DPOR).

The light-independent PChlide reductase plays an important role in the ability of anoxygenic photosynthetic bacteria, cyanobacteria, nonvascular plants, ferns and gymnosperms to form chlorophyll in

Table 1: Structural-functional features of NifH, ChlL, MinD, ArsA and CompA

\begin{tabular}{|c|c|c|c|c|c|}
\hline & $\mathrm{NifH}$ & ChlL & MinD & ArsA & CompA \\
\hline Localization & Cytoplasmic & Chloroplast & $\begin{array}{l}\text { Peripheral } \\
\text { membrane }\end{array}$ & $\begin{array}{l}\text { Extrinsic } \\
\text { membrane protein }\end{array}$ & Cytoplasm \\
\hline $\mathrm{O}_{2}$ sensitivity & Sensitive & Sensitive & Insensitive & Insensitive & Sensitive \\
\hline $\begin{array}{l}\text { Molecular } \\
\text { weight }\end{array}$ & $\sim 64 \mathrm{kDa}$ & $\sim 64 \mathrm{kDa}(?)$ & $\begin{array}{l}\sim 35 \mathrm{kDa} \\
\text { (monomer) }\end{array}$ & $\sim 63 \mathrm{kDa}$ & $\sim 54 \mathrm{kDa}$ \\
\hline Metal center & $4 \mathrm{Fe} 4 \mathrm{~S}$ & 4Fe4S (?) & -- & SbIII/AsIII & $4 \mathrm{Fe} 4 \mathrm{~S}$ \\
\hline Folding pattern & $\begin{array}{l}3 \text { layers: } \alpha / \beta / \alpha \\
\text { parallel or mixed } \\
\text { beta-sheets of } \\
\text { variable sizes }\end{array}$ & $\begin{array}{l}3 \text { layers: } \alpha / \beta / \alpha \\
\text { parallel or mixed } \\
\text { beta-sheets of } \\
\text { variable sizes (?) }\end{array}$ & $\begin{array}{l}3 \text { layers: } \alpha / \beta / \alpha \\
\text { parallel or mixed } \\
\text { beta-sheets } \\
\text { of variable sizes }\end{array}$ & $\begin{array}{l}3 \text { layers: } \alpha / \beta / \alpha \\
\text { parallel or mixed } \\
\text { beta-sheets of } \\
\text { variable sizes }\end{array}$ & $\begin{array}{l}3 \text { layers: } \alpha / \beta / \alpha \text {; } \\
\text { mixed beta-sheets of } \\
5 \text { strands, strand } 2 \text { is } \\
\text { antiparallel to others }\end{array}$ \\
\hline Dimerization & Homodimer & Homodimer (?) & $\begin{array}{l}\text { ATP dependent } \\
\text { dimerization }\end{array}$ & $\begin{array}{l}\text { Pseudodimer formed } \\
\text { by connection of ArsA } \\
1 \text { and ArsA } 2 \text { domains } \\
\text { through a short linker }\end{array}$ & Homodimer \\
\hline $\begin{array}{l}\text { Metal Center } \\
\text { Ligands }\end{array}$ & C 97, C 132 & C 95, C 129 & -- & $\begin{array}{l}\text { H } 148 \text { (A1), S } 420 \text { (A2) } \\
\text { C } 113 \text { (A1), C } 422 \text { (A2) } \\
\text { C } 172 \text { (A1), H } 453 \text { (A2) }\end{array}$ & C 127, C 166 \\
\hline $\begin{array}{l}\text { Nucleotide } \\
\text { binding domain }\end{array}$ & 10 GKGGIGKSTTT 20 & 7 GKGGIGKSTTS 17 & 9 GKGGVGKTTSS 19 & $\begin{array}{l}15 \text { GKGGIGKSSIS } 20 \text { (A1) } \\
334 \text { GKGGVGKTTMA } 344 \text { (A2) }\end{array}$ & $\begin{array}{l}7 \text { GIDVG } 11 \\
100 \text { VIDIG } 104\end{array}$ \\
\hline Switch I- & 39 DPIKADST 45 & 37 DPKHDST 43 & 43 DFDIGLRNL 51 & 45 DPASN 49 (I-A1) & 104 GGQD 107 \\
\hline Switch II & 125 DVLGDVV 131 & 122 DVLGDVV 128 & 120 DSPAG 124 & $\begin{array}{l}365 \text { DPAAG } 369 \text { (II-A1) } \\
142 \text { DTAPGHT } 149 \text { (I-A2) } \\
447 \text { DTAPGHY } 453 \text { (II-A2) }\end{array}$ & 127 CAAGTG 132 \\
\hline
\end{tabular}


Table 2: Percentage identity and percentage similarity scores (shown in parenthesis) between proteins having structural similarity to NifH

\begin{tabular}{lllllll}
\hline & NifH & ChlL & MinD & ArsA1 & ArsA2 & CompA \\
\hline NifH & $100 \%(100 \%)$ & $32 \%(63.8 \%)$ & $15.3 \%(52 \%)$ & $17.1 \%(53.7 \%)$ & $15 \%(48.2 \%)$ & $11 \%(49.6 \%)$ \\
ChlL & $32 \%(63.8 \%)$ & $100 \%(100 \%)$ & $17.7 \%(56.6 \%)$ & $14.3 \%(47.4 \%)$ & $15.6 \%(47.4 \%)$ & $14.7 \%(49.1 \%)$ \\
MinD & $15.3 \%(52 \%)$ & $17.7 \%(56.6 \%)$ & $100 \%(100 \%)$ & $17.7 \%(50 \%)$ & $18.1 \%(51.5 \%)$ & $11.5 \%(50.7 \%)$ \\
ArsA1 & $17.1 \%(53.7 \%)$ & $14.3 \%(47.4 \%)$ & $17.7 \%(50 \%)$ & $100 \%(100 \%)$ & $25 \%(61 \%)$ & $10 \%(46.6 \%)$ \\
ArsA2 & $15 \%(48.2 \%)$ & $15.6 \%(47.4 \%)$ & $18.1 \%(51.5 \%)$ & $25 \%(61 \%)$ & $100 \%(100 \%)$ & $12.2 \%(49.6 \%)$ \\
CompA & $11 \%(49.6 \%)$ & $14.7 \%(49.1 \%)$ & $11.5 \%(50.7 \%)$ & $10 \%(46.6 \%)$ & $12.2 \%(49.6 \%)$ & $100 \%(100 \%)$ \\
\hline
\end{tabular}

The scores were calculated using the ClustalW alignment program from the San Diego Supercomputer Center (SDSC) server. Identical residues within a pair of aligned proteins were used to compute the percentage identity; identical as well as similar residues were used to determine the percentage similarity

the dark ${ }^{[40,41]}$. Genetic and sequence analysis have suggested that DPOR consists of three protein subunits, ChlL, ChlB and ChlN as studied in cyanobacteria and higher photosynthetic forms, or BchL, BchN and BchB as studied in bacteria. Significant sequence similarity between the putative $\mathrm{BchL} / \mathrm{ChlL}, \mathrm{BchN} / \mathrm{ChlN}$ and $\mathrm{BchB} / \mathrm{ChlB}$ DPOR subunits has been found with the NifH, NifD and NifK subunits of nitrogenase respectively ${ }^{[40,42]}$. Amongst the group of NifH similar proteins being discussed herein, maximum similarity was found between NifH and ChlL structures, with an overall identity of $32 \%$ and a similarity of $\sim 64 \%$ (Table 2). Important features found conserved by comparison were the ATP-binding motif and the two Cys residues (C95 and C129) involved in coordinating the $[4 \mathrm{Fe}-4 \mathrm{~S}]$ cluster, in addition to two Asp residues postulated to have a role in ATP hydrolysis ${ }^{[43-45]}$, (Table 1), suggesting that the BchL/ChlL proteins might catalyze ATP-dependent transfer of electrons from a reductant such as ferredoxin, to a catalytic protein complex via the Fe:S center ${ }^{[14]}$.

Fujita and Bauer noted that based on the sequence similarity between DPOR and nitrogenase subunits, the BchL protein existed in solution as a dimer ${ }^{[14]}$. The reconstitution of the DPOR subunits showed that it consists of two separable components, the $\mathrm{BchL}$ protein and a $\mathrm{BchN}-\mathrm{BchB}$-protein complex, similar to that observed for nitrogenase, which has separable NifH and NifD-NifK components ${ }^{[14]}$.

Recently, the functional substitution for the chlL gene in Chlamydomonas reinhardtii was achieved by its replacement with the nifH gene of Klebsiella pneumoniae $^{[46]}$. The ability of NifH to functionally substitute for ChlL enhanced the view that the two proteins bear high structural equivalence to each other. Also, there are ongoing attempts towards substituting the nifH gene with the chlL gene and determining its sufficiency in the nitrogenase complex (Gavini et al., unpublished data). The presence of a [4Fe-4S] cluster in ChlL is an assumption based on its similarity to NifH.

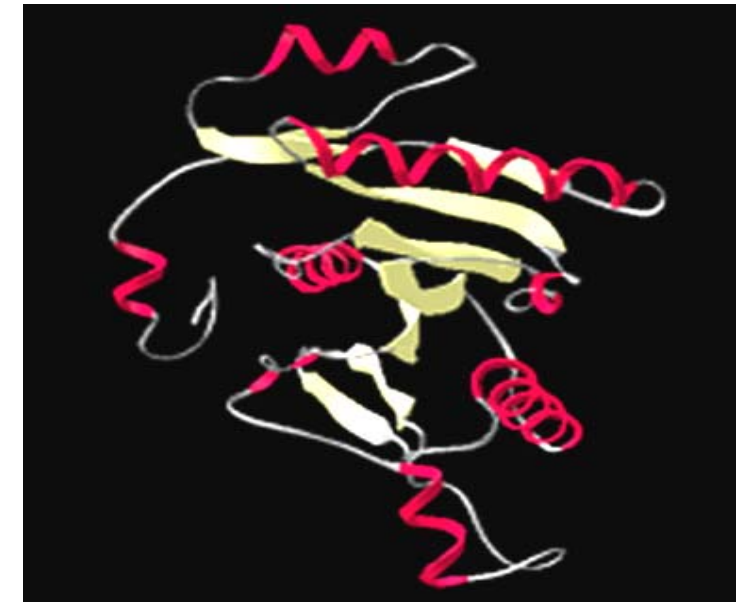

Fig. 3: A 3-D model of the ChlL protein ( $C$. reinhardtii) generated by using NifH (PDB ID: 1FP6) as a template. The monomeric ChlL model shows $\alpha$-helices colored in red and $\beta$ strands colored in light yellow.

The accessory gene products required for the mobilization of sulfur and iron for Fe-S cluster formation and for the activity and stabilization of NifH include NifS, NifU and NifM ${ }^{[47,48]}$. An inspection of the Chlamydomonas genome database for the equivalents of these accessory proteins did reveal their matches, leading to further evidence of a Fe-S cluster in ChlL ${ }^{[46]}$.

Inspite of many genetic and biochemical investigations of DPOR, the lack of a crystallized ChlL protein has deterred a detailed structural insight into the protein. As a step further to elucidate the structural aspects of the ChlL protein, we superimposed the protein sequence derived ChlL onto the NifH template (PDB ID: 1NIP), using the Swiss PDB (Deep View) protein modeling software (Fig. 3). The resultant ChlL protein model generated showed a marked resemblance to the NifH overall architecture and folds. As shown in Fig. 4, upon superimposition of a ChlL monomer on the 


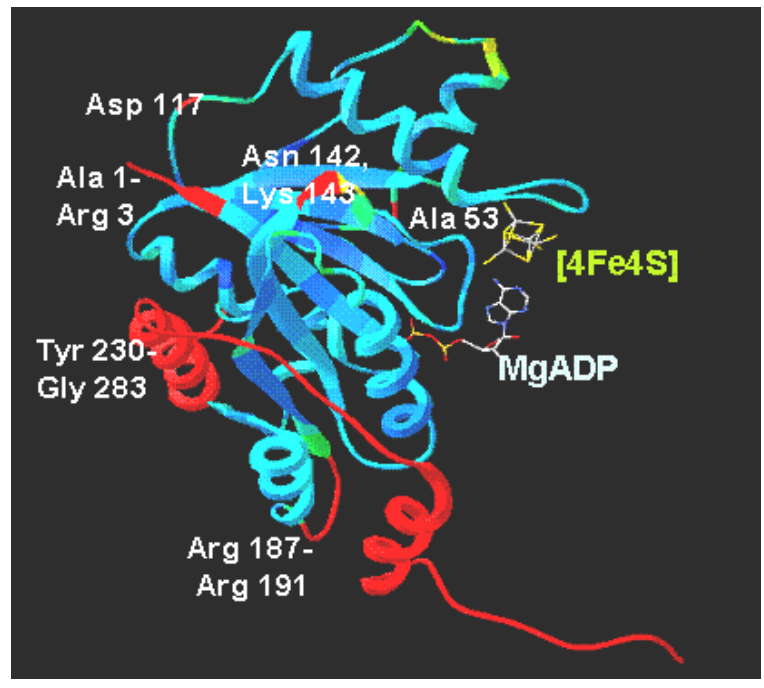

Fig. 4: NifH monomer structure colored according to differences with the ChlL model. The superimposition of ChlL on NifH indicate very few regions of dissimilarity (shown in red). A root mean square deviation type of coloration of the NifH protein (in comparison to ChlL) shows a particularly dissimilar 'tail' region (red stretch) spanning residues 230-289 of NifH. Each dissimilar region is indicated by the residues it is comprised of. The [4Fe-4S] cluster and the bound MgADP in NifH are indicated

$\mathrm{NifH}$ monomer, there was a wide overall structural alignment, however, the following residues in $\mathrm{NifH}$ appeared non-aligned: A1-R3, A53, D117, N142, K143, R187-R191, Y230-G283. Particularly, the tail region of $\mathrm{NifH}$, comprised of residues 230-283 (Fig. 4), differed extensively, since no such region of ChlL could be structurally aligned to it. Such absence of a tail region in ChlL could explain their phylogenetic divergence and possibly account for their functional differences.

Besides ChlL, CompA is another oxygen sensitive protein that has structural similarity to NifH and is a homodimer containing a [4Fe-4S] cluster Table 1. CompA shares $\sim 50 \%$ protein sequence similarity with NifH Table 2.

\section{CompA: GLUTAMATE DEGRADATION}

The hydroxyglutarate pathway, which involves the syn-elimination of water from (R)-2-hydroxyglutaryl$\mathrm{CoA}$ is key to glutamate degradation in Acidaminococcus fermentas ${ }^{[49,50]}$. This important step in the process of glutamate degradation is catalyzed by the enzyme 2-hydroxyglutaryl-CoA dehydratase. This enzyme is the product of the $h g d C A B$ genes and consists of the protein components, component $\mathrm{A}$ (CompA) and component B (CompD). CompA is an extremely oxygen-sensitive activator or initiator whereas CompD is moderately oxygen-sensitive and is the actual dehydratase. For activity of the enzyme, both protein components, $\mathrm{Mg}^{2+}$, ATP and a strong reducing agent are required. ATP is bound and hydrolyzed by CompA whilst the substrate binds to CompD ${ }^{[51]}$.

The X-ray crystallized structure of recombinant CompA protein from A. fermentas was determined at $3 \AA$ resolution by Locher et al. in $2001^{[19]}$. The CompA protein resembles an $\mathrm{L}$ shape, with its two arms being roughly similar in length. It has eight $\alpha$-helices and ten $\beta$-strands that form two flat sheets [19]. These two sheets meet at an angle of $60^{\circ}$ and the second sheet positions at the corner of the $\mathrm{L}$ and therefore is at the center of the protein. Helices 2 and 8, which are almost perpendicular to each other, fill up the large gap between the two $\beta$-sheets ${ }^{[19]}$. The ADP is bound between the two edges of the closely situated $\beta$-sheets. The $\beta$-phosphate of the ADP is positioned $19 \AA$ from the $[4 \mathrm{Fe}-4 \mathrm{~S}]$ cluster that lies at the dimeric interface of CompA $^{[19]}$. The nucleotide binding pocket in CompA is formed of residues in strands 1 and 6 , containing the Gly7-X-Asp-X-Gly11 and the Val100-X-Asp-XGly104 motif (Table 1), as found in sugar kinases ${ }^{[52]}$.

Though NifH and CompA are from unrelated phylogenetic families, several structural resemblances exist between them. Like NifH, CompA is a homodimeric enzyme (54 kDa), with one ADP bound to each subunit and a single $[4 \mathrm{Fe}-4 \mathrm{~S}]$ cluster at its dimeric interface. The [4Fe-4S] cluster in both, CompA and NifH, are solvent exposed $\left(\sim 20 \AA^{2}\right)$ instead of buried, as observed in other [4Fe-4S] cluster containing proteins $^{[19,53]}$. Excluding the cluster, the interface between CompA monomers accounts for only $\sim 800 \AA^{2}$ buried area ${ }^{[19]}$. From predictions based on protein sequence comparisons and subsequent structural analysis of the X-ray crystallized CompA, the [4Fe-4S] cluster was found to be coordinated by two cysteine residues, C 127 and 166, provided by each monomer ${ }^{[19]}$. The coordination of the Cys ligands with the [4Fe-4S] cluster in NifH and CompA is shown in Fig. 5A and 5B respectively. Two helices, each from one subunit, pointing with their N-termini towards the [4Fe-4S] cluster and forming a helix-cluster-helix angle of $105^{\circ}$, is a remarkable feature of Comp $\mathrm{A}^{[54]}$. Such similar architecture has also been noted in $\mathrm{NifH}$, revealing a helix-cluster-helix angle of $150^{\circ[55]}$. Further, as observed in the NifH complex with MoFe protein in the 

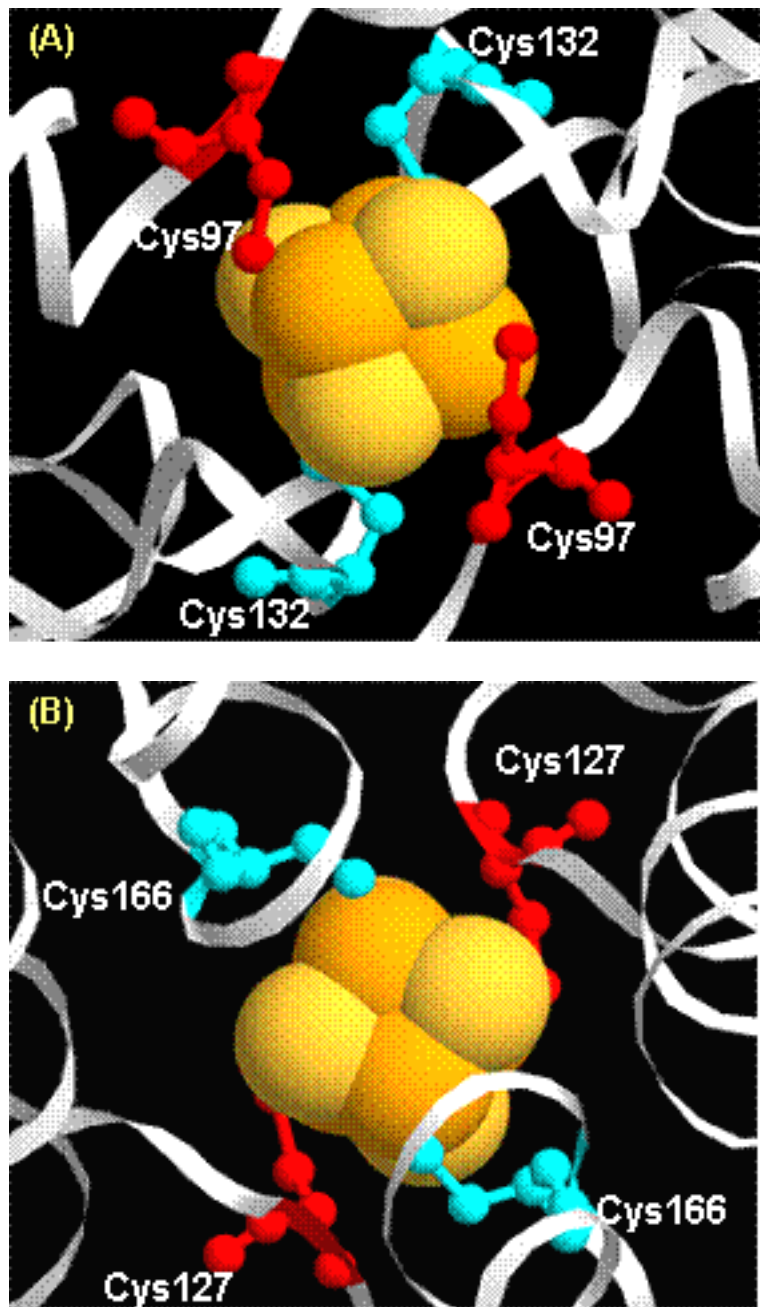

Fig. 5: Similar view of [4Fe-4S] cluster bound by Cys ligands in NifH and CompA. (a): In NifH, Cys 97 (ball and stick- red) and Cys 132 (ball and stick- cyan) serve as ligands for the [4Fe-4S] cluster (spacefill-CPK) (b): In CompA, Cys 127 ( ball and stick- red) and Cys 166 (ball and stick- cyan) serve as ligands for the [4Fe-4S] cluster (spacefill-CPK)

presence of $\mathrm{ADP}-\mathrm{AlF}_{4}^{-}$, the CompA also probably opens to an angle of $180^{\circ}$ upon ATP binding. This open structure then resembles an archer shooting arrows, wherein the helix-cluster-helix with two bound ADP could be superimposed on the string of the archers bow, hence leading to the term archerase for Comp $\mathrm{A}^{[56]}$.

The Switch II region in NifH includes the Cys132 residue that coordinates the $[4 \mathrm{Fe}-4 \mathrm{~S}]$ cluster and consequently links the nucleotide and the cluster in $\mathrm{NifH}^{[57]}$. The Switch II region interacts with the $\gamma$ -

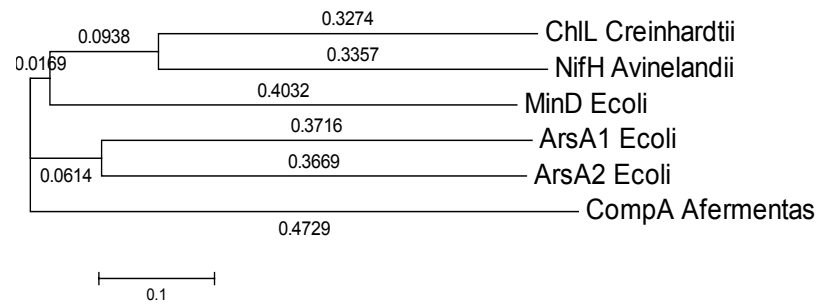

Fig. 6: Evolutionary distances between NifH-like proteins. The tree is based on the protein sequence alignment of the $\mathrm{NifH}$ ( $A$. vinelandii), MinD (E. coli), ChlL (C. reindhardtii), ArsA1 (E. coli), ArsA2 (E. coli) and CompA (A. fermentas) proteins and constructed using the neighbor-joining method. The distances based on percentage divergence are mentioned alongwith each branch

phosphate of the nucleotide and upon hydrolysis changes its conformation to the nucleoside diphosphate state. Thus, in NifH, the regulation of electron transfer by protein conformational change is directed by the nucleotide hydrolysis. In CompA, a structural analogue equivalent to the NifH Switch II region has been mapped to the residues 104 to 107 and 127 to 132 , positioned between the ADP and [4Fe-4S] cluster; these segments connect the nucleotide-binding site to the cluster and are most likely involved in coupling ATP hydrolysis to the changes in the cluster-binding region $^{[19]}$.

Based on the evolutionary distances, CompA appears to have diverged from $\mathrm{NifH}$ at a much earlier time than ChlL, MinD and ArsA (Fig. 6). MinD and ArsA are both oxygen insensitive and bear highly similar structural folds to NifH (Table 1). Within this group of NifH similar proteins (Table 1), MinD and ArsA are marked by the absence of the $[4 \mathrm{Fe}-4 \mathrm{~S}]$ cluster. The ArsA protein could be considered to be a dimer of two NifH like subunits ArsA1 and ArsA2; however only a monomeric form of MinD has been available for study by researchers, eventhough there is evidence for ATP dependent dimerization of $\mathrm{MinD}^{[12]}$.

\section{MinD: SPATIAL REGULATOR OF CELL DIVISION}

The peripheral membrane ATPase MinD, along with MinC and MinE, is a component of the Min system responsible for correct placement of the division site in E. coli cells ${ }^{[58]}$. MinD helps in blocking unnecessary septation events at the poles by rapidly migrating from one cell pole to the other. The binding 
of MinE to MinD induces hydrolysis of ATP and the release of MinD into the cytoplasm ${ }^{[59]}$. The ATP binding cycle induced by MinE results in the rapid movement of MinD from one cell pole to the opposite cell pole, forming alternating broad polar zones ${ }^{60]}$. It was recently reported that MinC and MinD, which were previously thought to be diffusely associated with the membrane at the poles, were in reality organized into extended spirals that coiled around the cell ${ }^{[61]}$. Although it has been shown that MinD dimerizes in the presence of ATP, only its monomeric form with ADP, AMPPCP or without a nucleotide has been crystallized till now ${ }^{[18,62,63]}$. These crystallized MinD were obtained from three different archaeal species, Pyrococcus horikoshii OT3, Pyrococcus furiosus, Archaeoglobus fulgidus based on the relative ease of their crystallization. The MinD molecule from $P$. horikoshii was found to consist of a $\beta$-sheet comprised of 7 parallel and 1 antiparallel strands and 11 peripheral $\alpha$ helices $^{[63]}$. Though NifH and MinD share only $\sim 16 \%$ identical residues (Table 2), the overall folding topology of the two proteins are clearly related to each other ${ }^{[63]}$.

Since the dimeric form of crystallized MinD was unavailable, Lutkenhaus and Sundaramoorthy used the $\mathrm{NifH}$ structure as a template for modeling a dimeric $\mathrm{MinD}^{[11]}$. We attempted a similar structural alignment for this review, using the crystallized MinD (PDB ID: 1HYQ) and NifH (PDB ID: 1NIP) structures as shown in Fig. 7. The superimposition of two MinD monomers (green and blue) on a NifH dimer template (yellow) revealed that other than certain loops at the surface of the two proteins, an additional loop and an $\alpha$-helix present near the C-terminus in $\mathrm{NifH}$, the superimposition yielded a good fit, similar to the observation of Lutkenhaus and Sundaramoorthy [11]. Likewise, Hayashi et al. found that NifH and MinD exhibited similar architectural regions in their ATPase and GTPase domains $(\beta 7, \beta 6, \beta 1, \beta 5, \beta 2, \beta 4, \beta 3$, the Ploop, $\alpha 1, \alpha 7, \alpha 8$ ) yielding $1.2 \AA$ r.m.s.d. value for $81 \mathrm{C}$ $\alpha$ atoms $^{[18]}$. Both NifH and MinD contain the deviant Walker motif A [X-K-G-G-X-X-K-(T/S) $]^{[21]}$. It was however observed that the C97 and C132 ligands of the [4Fe-4S] cluster of NifH were not conserved in $P$. furiosus MinD. Upon protein sequence comparison of NifH and MinD from A. fulgidus, a distinct presence of the Fer4_NifH conserved domain, typically found in [4Fe-4S] iron-sulfur cluster binding proteins and $\mathrm{NifH} / \mathrm{frxC}$ protein families, was detected in $\mathrm{MinD}^{[64]}$, spanning residues 133-240 of MinD that correspond to residues $144-251$ of $\mathrm{NifH}$.

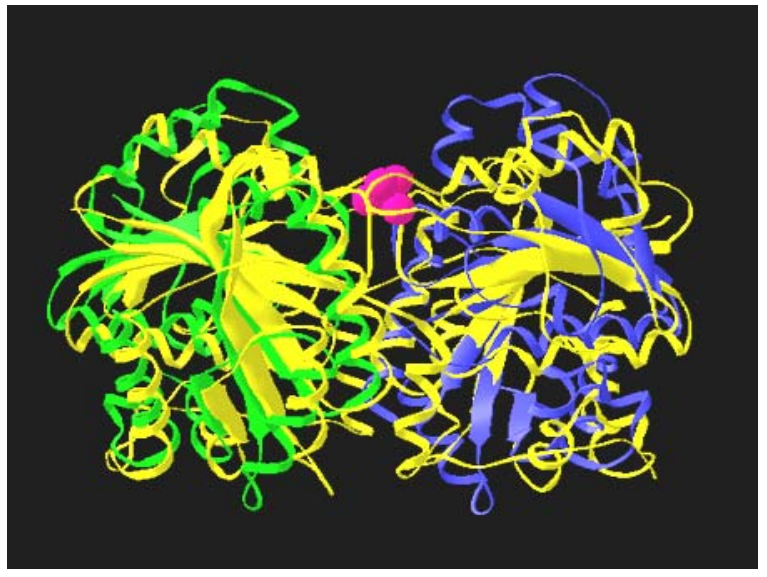

Fig. 7: Comparisons between $\mathrm{NifH}$ and MinD. The MinD monomers (blue and green) have been superimposed on a NifH dimer (yellow). The [4Fe-4S] cluster of $\mathrm{NifH}$ is shown as a spacefilled body (magenta)

\section{ArsA: OXYANION TRANSLOCATING ATPASE SUBUNIT}

The ArsA protein that is the catalytic subunit of the $\mathrm{As}(\mathrm{III}) / \mathrm{Sb}$ (III)-translocating ArsAB complex, has long been speculated to be a distant relative of $\mathrm{NifH}^{[13]}$. The ArsB subunit serves as a membrane anchor for ArsA and forms the anion-translocating sector of the ArsAB pump $^{[65]}$. The crystallized structure of the ArsA protein $^{[20]}$ has revealed many structural details that establish its similarity to the NifH protein. ArsA is a 583-residue polypeptide that has homologous $\mathrm{N}$ terminal (A1) and C-terminal (A2) halves, indicating an evolutionary gene duplication and fusion ${ }^{[66]}$. This $63 \mathrm{kDa}$ ATPase contains two consensus nucleotidebinding motifs, one each in the A1 and A2 halves. The $\mathrm{A} 1$ and $\mathrm{A} 2$ halves are held together by a short linker peptide ${ }^{[67]}$. ArsA, like NifH exists in a more open conformation in the presence of ADP and contains two NBSs. The two NBSs are each filled with MgADP and lie at the interface between A1 and A2. In each NBS, $\mathrm{Mg}^{2+}$ is octahedrally coordinated by the $\beta$-phosphate of ADP, several water molecules and a threonine hydroxyl. When compared to D39 and D129 residues of the NifH protein that play a role in the MgATP hydrolysis, D45 from the ArsA1 NBS directly coordinates with $\mathrm{Mg}^{2+}$ whereas D364 of the ArsA2 NBS coordinates indirectly to it via a water molecule $^{[20,35,68]}$.

ArsA is a homodimer in its catalytically active form $^{[69]}$ and dimerization is favored by the formation of a three-coordinate complex among three specific 
cysteine thiolates (Cys-113, Cys-172 and Cys-442) and the effector, $\mathrm{Sb}$ (III) or $\mathrm{As}(\mathrm{III})^{[70,71]}$. Each $\mathrm{Sb}$ (III) is coordinated by one $\mathrm{A} 1$ residue and one $\mathrm{A} 2$ residue. These residues include His148 (A1) and Ser420 (A2); Cys113 (A1) and Cys422 (A2); Cys172 (A1) and His453 (A2). The oxygen insensitivity of ArsA in comparison to NifH could most reasonably be assigned to the difference in the metal center of both these proteins.

ArsA is twice the size of NifH, yet since it consists of two similar domains connected by a short linker, each ArsA monomer could actually be considered to be a pseudodimer, as regarded herein. Information obtained on ArsA (PDB ID: 1IHU) and NifH (PDB ID: $1 \mathrm{G} 5 \mathrm{P})$ protein families from the SCOP database ${ }^{[22]}$ and their comparison based on structural modeling made it evident that the core $\beta$-sheet pattern in both proteins, made up of at least 7 parallel $\beta$-strands, could fit with minimum root square deviation upon superimposition (Fig. 8A). It was also observed that the Cys97 ligands for the [4Fe-4S] cluster from the two NifH monomers could be aligned to the Cys 113 and Cys422 residues of ArsA1 and ArsA2 respectively (Fig. 8B). Moreover, the Cys132 ligands for the [4Fe-4S] cluster of $\mathrm{NifH}$ corresponded to the His 148 (A1) and His453 (A2) of the antimony site. The superimposition of the ArsA1 or ArsA2 with a NifH monomer revealed a clear coincidence of the metalloid center of ArsA and the [4Fe-4S] cluster of NifH. Furthermore, the $\mathrm{D}^{142 / 447}$ TAPTGH $^{148 / 453}$ signature sequence of ArsA1 and ArsA2 $2^{[20]}$ has an exact counterpart in $\mathrm{NifH}$ and is believed to correspond to the Switch II region of Gproteins $^{[37]}$. The comparison of the residues of ArsA1 and ArsA2 corresponding to the region of $\mathrm{NifH}$ involved in complex formation with MoFe (NifDK) protein yielded a similar patch of acidic residues. E112 of NifH and K400 of the NifDK protein which specifically crosslink during this process are found within a pocket of other close acidic residues and basic residues respectively ${ }^{[17]}$. E112 in NifH is surrounded by multiple carboxylic acids, including E68, 71, 73, 110, 111, 112, 116 and D69 ${ }^{[17]}$. Although E112 is highly specific for the crosslinking, it is not a conserved residue among the NifH protein sequences. However, the acidic patch surrounding it is well conserved. The ArsA2 region comprising of residues from L398 to G439, when compared with the region G94 to F135 of $\mathrm{NifH}$, revealed eight glutamate residues, two of which had exact counterparts in $\mathrm{NifH}$ and E112 of $\mathrm{NifH}$ corresponded to D417 of ArsA2 (Fig. 9A). The buried interface between the A1 and A2 domains of ArsA was found to be relatively small $(<10 \%)$, compared with the total surface of the enzyme ${ }^{[20]}$, rendering ArsA as a hollow protein with a large central cavity (Fig. 9B). The hinge region between the ArsA1 ArsA2 polypeptides is formed by a 25-residue linker peptide in the region of $289-313^{[67]}$. The structural comparison of the linker region of ArsA by superimposition on NifH indicated the absence of such a stretch in NifH.
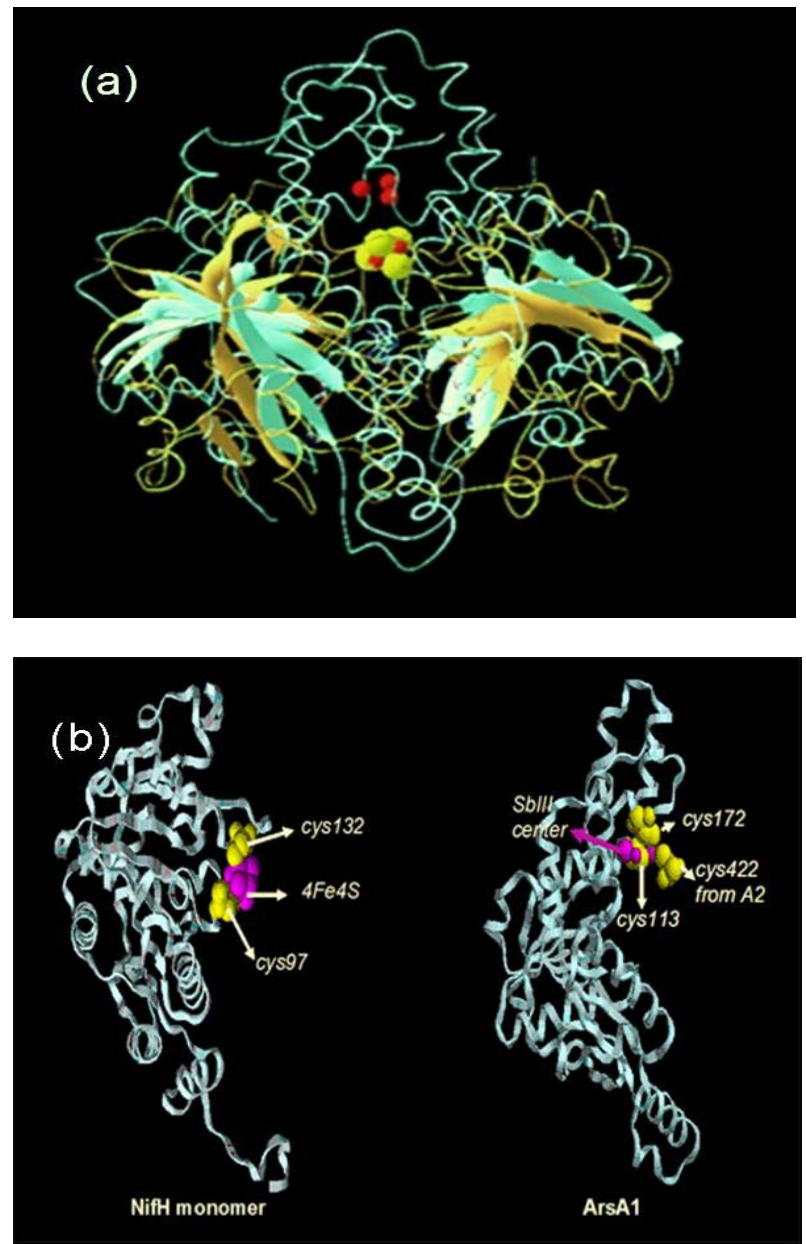

Fig. 8: Structural comparisons between ArsA and NifH. (a): Superimposition of ArsA (cyan) on $\mathrm{NifH}$ (yellow) shows near coincidence of their metal clusters and a good fit of the $\beta$-strand structures. [4Fe-4S] cluster of $\mathrm{NifH}$ shown spacefilled in yellow and $\mathrm{Sb}$ (III)/As(III) metal center is spacefilled in red (b): A comparison of the metal centers and ligands of NifH and ArsA. Both protein structures are represented as blue ribbons and the metal centers are shown in magenta (spacefill). C97 and 132 for NifH and $\mathrm{C} 113$ and $\mathrm{C} 172$ for ArsA2 are indicated by arrows. Also C422 implicated as a ligand from ArsA1 is indicated 
(a)

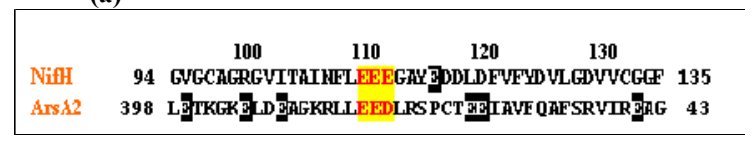

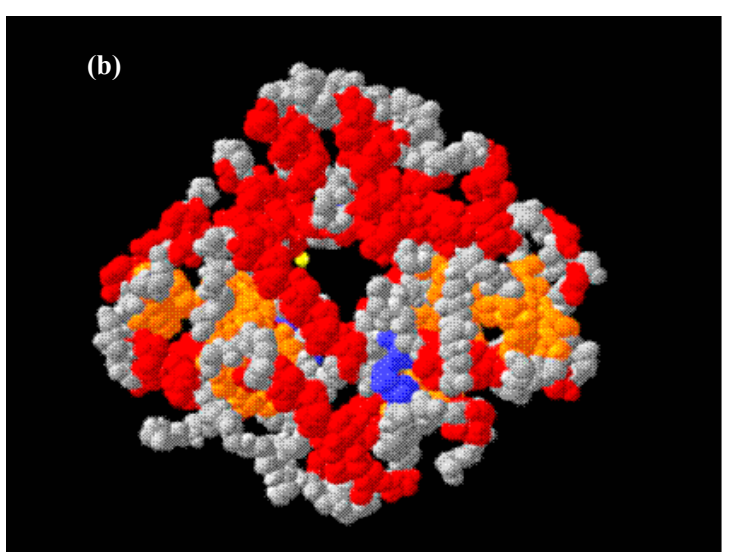

Fig. 9: (a): Protein sequence alignment of $\mathrm{NifH}$ and ArsA2 in the region spanning of and corresponding to important residues in $\mathrm{NifH}$ required for complex formation with the MoFe protein (Gly 94 to Phe 135 in NifH and Leu 398 to Gly 439 in ArsA). An acidic patch can be observed in ArsA, indicated by the presence of eight glutamate residues (highlighted in black). Glu 112 of $\mathrm{NifH}$ corresponds to Asp 417 of ArsA2. Asp 417 could be also considered as Asp 99 when ArsA2 is taken as a single unit. (b): The ArsA is a hollow protein with a central cavity as seen in this view. The protein is completely spacefilled and $\alpha$-helices are in red, $\beta$-strands are in orange, coils in gray and part of the metal center seen is in yellow

Strong evidence for the functional conservation of essential structural domains in NifH and ArsA was recently obtained by substitution of the ArsA2 half of ArsA by NifH, in E. coli ${ }^{[72]}$. The positive complementation suggested that these proteins were either derived from a common ancestral protein or that one of these was the actual precursor protein from which the other has evolved by divergence. The study also emphasized the importance of the conservation of the group of motifs present in both proteins that allowed NifH to substitute for the function of ArsA2.

\section{EVOLUTIONARY RELATIONSHIPS}

The structural similarities between $\mathrm{NifH}$ and the proteins ArsA, MinD, CompA and ChlL led to the question that whether all these proteins have diverged from a single closely related ancestor or from one of the proteins amongst this group. Computation of the phylogenetic distances between $\mathrm{NifH}$ and these proteins suggested that $\mathrm{ChlL}$ and MinD were most closely related to $\mathrm{NifH}$ whereas ArsA and CompA were relatively more distant from $\mathrm{NifH}$.

In order to visualize the relationships between these structurally related proteins, a phylogram based on their amino acid sequence alignment was generated (Fig. 6). Due to the functional diversity of the proteins, direct evolutionary interpretations should be avoided but as shown in Fig. 6, there exist three broad divisions that phylogenetically classify the polypeptides NifH, ArsA1, ArsA2, ChlL, MinD and CompA. The phylogenetic tree suggests that $\mathrm{NifH}$ and ChlL share maximum similarities and have probably diverged most recently compared to others. It also suggests that both $\mathrm{NifH}$ and ChlL could have evolved from a MinD ancestor and that ArsA1, ArsA2 and CompA, though structurally similar to $\mathrm{NifH}$, may have diverged farther away during evolution.

\section{ACKNOWLEDGMENTS}

This research is partly supported by NIH and NSF to NG and LP. This material was also based on work supported by the National Science Foundation, while NG working at the Foundation. We thank members of Gavini/Pulakat laboratory for helpful discussions.

\section{REFERENCES}

1. Rees, D.C., F.A. Tezcan, C.A. Haynes, M.Y. Walton, S. Andrade, O. Einsle and J.B. Howard, 2005. Structural basis of biological nitrogen fixation. Philos. Transact. A Math. Phys. Eng. Sci., 363: 971-984.

2. Rees, D.C, 2002. Great metalloclusters in enzymology. Ann. Rev. Biochem., 71: 221-246.

3. Seefeldt, L.C. and D.R. Dean, 1997. Role of nucleotides in nitrogenase catalysis. Acc. Chem. Res., 30:260-266.

4. Burgess, B.K. and D.J. Lowe, 1996. Mechanism of molybdenum nitrogenase. Chem. Rev., 96: 2983-3011.

5. Georgiadis, M.M., H. Komiya, P. Chakrabarti, D. Woo, J.J. Kornuc and D.C. Rees, 1992. Crystallographic structure of the nitrogenase iron protein from Azotobacter vinelandii. Sci., 257: 1653-1659.

6. Chen, L., N. Gavini, H. Tsuruta, D. Eliezer, B.K. Burgess, S. Doniach and K.O. Hodgson, 1994. MgATP-induced conformational changes in the iron protein from Azotobacter vinelandii, as studied by small-angle x-ray scattering. J. Biol. Chem., 269: 3290-3294. 
7. Tal, S., T.W. Chun, N. Gavini and B.K. Burgess, 1991. The delta nifB (or delta nifE) FeMo cofactordeficient MoFe protein is different from the delta nifH protein. J. Biol. Chem., 266: 10654-10657.

8. Gavini, N. and B.K., 1992. FeMo cofactor synthesis by a nifH mutant with altered MgATP reactivity. J. Biol. Chem., 267: 21179-21186.

9. Ma, L., N. Gavini, H.I. Liu, B. Hedman, K.O. Hodgson and B.K. Burgess, 1994. Large scale isolation and characterization of the molybdenumiron cluster from nitrogenase. J. Biol. Chem., 269: 18007-18015.

10. Gavini, N., L. Ma, G. Watt and B.K. Burgess, 1994. Purification and characterization of a FeMo cofactor-deficient $\mathrm{MoFe}$ protein. Biochemistry, 33: 11842-11849.

11. Lutkenhaus, J. and M. Sundaramoorthy, 2003. MinD and role of the deviant Walker A motif, dimerization and membrane binding in oscillation. Mol. Microbiol., 48: 295-303.

12. Hu, Z., C. Saez and J. Lutkenhaus, 2003. Recruitment of MinC, an inhibitor of Z-ring formation, to the membrane in E. coli: Role of MinD and MinE. J. Bacteriol., 185: 196-203.

13. Gatti, D., B. Mitra and B.P. Rosen, 2000. Escherichia coli soft metal ion-translocating ATPases. J. Biol. Chem., 275: 34009-34012.

14. Fujita, Y. and C.E. Bauer, 2000. Reconstitution of light-independent protochlorophyllide reductase from purified $\mathrm{BchL}$ and $\mathrm{BchN}-\mathrm{BchB}$ subunits. In vitro confirmation of nitrogenase likfe features of a bacteriochlorophyll biosynthesis enzyme. J. Biol. Chem., 275: 23583-23588.

15. de Boer, P.A.J., R.E. Crossley and L.I. Rothfield, 1992. Roles of MinC and MinD in the site-specific septation block mediated by the MinCDE system of Escherichia coli. J. Bacteriol., 174: 63-70.

16. Fujita, Y., Y. Takahashi, T. Kochi, H. Ozeki, K. Ohyama and H. Matsubara, 1989. Identification of a novel NifH-like $(f r x C)$ protein in chloroplasts of the Liverwort Marchantia polymorpha. Plant. Mol. Biol., 13: 551-561.

17. Schmid, B., O. Einsle, H.J. Chiu, A. Willing, M. Yoshida, J.B. Howard and D.C. Rees, 2002. Biochemical and structural characterization of the cross-linked complex of nitrogenase: Comparison to the ADP-AlF ${ }_{4}^{-}$-stabilized structure. Biochem., 41: 15557-15565.

18. Hayashi, I., T. Oyama and K. Morikawa, 2001. Structural and functional studies of MinD ATPase: Implications for the molecular recognition of the bacterial cell division apparatus. EMBO. J., 20: 1819-1828.
19. Locher, K.P., M. Hans, A.P. Yeh, B. Schmid, W. Buckel and D.C. Rees, 2001. Crystal structure of the Acidaminococcus fermentans 2hydroxyglutaryl-CoA dehydratase component A. J. Mol. Biol., 307: 297-308.

20. Zhou, T., S. Radaev, B.P. Rosen and D.L. Gatti, 2000. Structure of the ArsA ATPase: The catalytic subunit of a heavy metal resistance pump. EMBO. J., 19: 4838-4845.

21. Koonin, E.V., 1993. A superfamily of ATPases with diverse functions containing either classical or deviant ATP-binding motif. J. Mol. Biol., 229: 1165-1174.

22. Murzin, A.G., S.E. Brenner, T. Hubbard and C. Chothia, 1995. SCOP: A structural classification of proteins database for the investigation of sequences and structures. J. Mol. Biol., 247: 536-540.

23. Finkelstein, A.V., A.M. Gutun and A.Y. Badretdinov, 1993. Why are the same protein folds used to perform different functions? FEBS Lett., 325: 23-28.

24. Schlessman, J.L., D. Woo, L. Joshua-Tor, J.B. Howard and D.C. Rees, 1998. Conformational variability in structures of the nitrogenase iron proteins from Azotobacter vinelandii and Clostridium pasteurianum. J. Mol. Biol., 280: 669-685.

25. Hausinger, R.P. and J.B. Howard, 1983. Thiol reactivity of the nitrogenase Fe-protein from Azotobacter vinelandii. J. Biol. Chem., 258: 1348613492.

26. Howard, J.B. and D.C. Rees, 1996. Structural basis of biological nitrogen fixation. Chem. Rev., 96: 2965-2982.

27. Seefeldt, S.C. and L.E. Mortenson, 1993. Increasing nitrogenase catalytic efficiency for MgATP by changing serine 16 of its Fe protein to threonine: Use of $\mathrm{Mn}(2+)$ to show interaction of serine 16 with $\mathrm{Mg}(2+)$. Protein Sci., 2: 93-102.

28. Milner-White, E.J., J.R. Coggins and I.A. Anton, 1991. Evidence for an ancestral core structure in nucleotide-binding proteins with the type-A motif. J. Mol. Biol., 221: 751-754.

29. Schulz, G.E, 1992. Binding of nucleotides by proteins. Curr. Opin. Struct. Biol., 2: 61-67.

30. Sprang, S.R., 1997. G-protein mechanisms: insights from structural analysis. Annu. Rev. Biochem., 66: 639-678.

31. Walker, J.E., M. Saraste, M.J. Runswick and N.J. Gay, 1982. Distantly related sequences in the alpha- and beta-subunits of ATP synthase, myosin, kinases and other ATP-requiring enzymes and a common nucleotide binding fold. EMBO. J., 8: 945-981. 
32. Seefeldt, L.C., T.V. Morgan, D.R. Dean and L.E. Mortenson, 1992. Mapping the site(s) of magnesium-ATP and magnesium-ADP interaction with the nitrogenase of Azotobacter vinelandii. Lysine 15 of the iron protein plays a major role in magnesium-ATP interaction. J. Biol. Chem., 267: 6680-6688.

33. Wolle, D., D.R. Dean and J.B. Howard, 1992. Nucleotide-iron-sulfur cluster signal transduction in the nitrogenase iron-protein: the role of Asp125. Sci., 258: 992-995.

34. Ryle, M.J., W.N. Lanzilotta, L.E. Mortenson, G.D. Watt and L.C. Seefeldt, 1995. Evidence for a central role of lysine 15 of Azotobacter vinelandii nitrogenase iron protein in nucleotide binding and protein conformational changes. J. Biol. Chem., 270:13112-13117.

35. Lanzilotta, W. N., M. J. Ryle, L. C. Seefeldt, 1995 Nucleotide hydrolysis and protein conformational changes in Azotobacter vinelandii nitrogenase iron protein: defining the function of aspartate 129 . Biochem., 34: 10713-10723.

36. Jang, S.B., M.S. Jeong, L.C. Seefeldt and J.W. Peters, 2004. Structural and biochemical implications of single amino acid substitutions in the nucleotide-dependent switch regions of the nitrogenase Fe protein from Azotobacter vinelandii. J. Biol. Inorg. Chem., 9:1028-1033.

37. Jang, S.B., L.C. Seefeldt and J.W. Peters, 2000. Insights into nucleotide signal transduction in nitrogenase: Structure of an iron protein with MgADP bound. Biochem., 39: 14745-14752.

38. Tezcan, F.A., J.T. Kaiser, D. Mustafi, M.Y. Walton, J.B. Howard and D.C. Rees, 2005. Nitrogenase complexes: Multiple docking sites for a nucleotide switch protein. Sci., 309: 1377-1380.

39. Christiansen, J., J.M. Chan, L.C. Seefeldt and D.R. Dean, 2000. The role of the MoFe protein alpha-125Phe and beta-125Phe residues in Azotobacter vinelandii $\mathrm{MoFe}$ protein-Fe protein interaction. J. Inorg. Biochem., 80: 195-204.

40. Fujita, Y., 1996. Protochlorophyllide reduction: A key step in the greening of plants. Plant Cell Physiol., 37: 411-421.

41. Godje, O., M. Peyerl, T. Seebauer, P. Lamm, H. Mair, B. Reichart and G.A. Armstrong, 1998. Greening in the dark: Light independent chlorophyll biosynthesis from anoxygenic photosynthetic bacteria to gymnosperms. J. Photochem. Photobiol., B 43: 87-100.

42. Fujita, Y., H. Matsumoto, Y. Takahashi and H. Matsubara, 1993. Identification of a nifDK-like gene (ORF467) involved in the biosynthesis of chlorophyll in the cyanobacterium Plectonema boryanum. Plant Cell Physiol., 34: 305-314.
43. Suzuki, J.Y. and C.E. Bauer, 1992. Lightindependent chlorophyll biosynthesis: Involvement of the chloroplast gene chlL (frxC). Plant Cell, 4: 929-940.

44. Huang, C. and X.Q. Liu, 1992. Nucleotide sequence of the $\operatorname{fr} x C$, pet $B$ and $\operatorname{trn} L$ genes in the chloroplast genome of Chlamydomonas reinhardtii. Plant Mol. Biol., 18: 985-988.

45. Burke, D.H., J.E. Hearst and A. Sidow, 1993. Early evolution of photosynthesis: clues from nitrogenase and chlorophyll iron proteins. Proc. Natl. Acad. Sci. USA, 90: 7134-7138.

46. Cheng, Q., A. Day, M. Dowson-Day, G-F. Shen and R. Dixon, 2005. The Klebsiella pneumoniae nitrogenase $\mathrm{Fe}$ protein gene (nifH) functionally substitutes for the chlL gene in Chlamydomonas reinhardtii. Biochem. Biophys. Res. Commun., 329: 966-975.

47. Gavini, N. and L. Pulakat, 2002. Role of NifM in Maturation of the Fe Protein of Nitrogenase In: Nitrogen Fixation: Global Perspectives, Finan, T., M. OBrian, D. Layzell, K. Vessey and Newton B. Wallingford (Eds.), Oxford: CABI International, pp: 228-232.

48. Lill, R., U. Muhlenhoff, 2005. Iron-sulfur-protein biogenesis in eukaryotes. Trends. Biochem. Sci., 30: 133-141.

49. Buckel, W., 1980. The reversible dehydratation of $(R)$-2-hydroxyglutarate to $(E)$ glutaconate. Eur. J. Biochem., 106: 439-447.

50. Muller, U. and W. Buckel, 1995. Activation of $(R)-$ 2-hydroxyglutaryl-CoA dehydratase from Acidaminococcus fermentas. Eur. J. Biochem., 230: 698-704.

51. Hans, M., J. Sievers, U. Muller, J. A. Vorholt, D. Linder and W. Buckel, 1999. hydroxyglutaryl-CoA dehydratase from Clostridium symbiosum. Eur. J. Biochem., 265: 404-414.

52. Hurley, J.H., 1996. The sugar kinase/heat shock protein 70/ actin superfamily: implications of conserved structure for mechanism. Annu. Rev. Biophys. Biomol. Struct., 25: 137-162.

53. Strop, P., P.M. Takahar, H.J. Chiu, C. Hayley, C. Angrove, B.K. Burgess and D.C. Rees, 2001. Crystal structure of the all-ferrous $[4 \mathrm{Fe}-4 \mathrm{~S}]^{0}$ form of the nitrogenase iron protein from Azotobacter vinelandii. Biochem., 40: 651-656.

54. Kim, J., M. Hetzel, C.D. Boiangiu and W. Buckel, 2004. Dehydratation of $(R)$-2-hydroxyacyl-CoA to enoyl-CoA in the fermentation of $\alpha$-amino acids by anaerobic bacteria. FEMS. Microbiol. Rev., 28: 455-468. 
55. Howard, J.B. and D.C. Rees, 1994. Nitrogenase: A nucleotide-dependent molecular switch. Annu. Rev. Biochem., 63: 235-264.

56. Buckel, W., 2003. Archerasen- eine wachsende Enzymfamilie katalysiert ATP-induzierten elektronentransport. BIOspektrum, 9: 146-149.

57. Schindelin, H., C. Kisker, J.L. Schlessman, J.B. Howard and D.C. Rees, 1997. Structure of $\mathrm{ADP}-\mathrm{AlF}_{4}{ }^{-}$stabilized nitrogenase complex and its implications for signal transduction. Nature, 387: 370-376.

58. Rothfield, L.I., Y.L. Shih and G. King, 2001. Polar explorers: Membrane proteins that determine division site placement. Cell, 106: 13-16.

59. Hu, Z., E.P. Gogol and J. Lutkenhaus, 2002. Dynamic assembly of MinD on phospholipid vesicles regulated by ATP and MinE. Proc. Natl. Acad. Sci., 99: 6761-6766.

60. Raskin, D.M. and P.A. de Boer, 1999. Rapid poleto-pole oscillation of a protein required for directing division to the middle of Escherichia coli. Proc. Natl. Acad. Sci., 96: 4971-4976.

61. Shih, Y.L., T. Le and L. Rothfield, 2003. Division site selection in Escherichia coli involves dynamic redistribution of Min proteins within coiled structures that extend between the two cell poles. Proc. Natl. Acad. Sci., 100: 7865-7870.

62. Cordell, S.C. and J. Lowe, 2001. Crystal structure of the bacterial cell division regulator MinD. FEBS Lett., 492: 160-165.

63. Sakai, N., M. Yao, H. Itou, N. Watanabe, F. Yumoto, M. Tanokura and I. Tanaka, 2001. The three-dimensional structure of septum sitedetermining protein MinD from Pyrococcus horikoshii OT3 in complex with MgADP. Structure (Camb), 9: 817-826.

64. Marchler-Bauer, A., J.B. Anderson, P.F. Cherukuri, C. DeWeese-Scott, L.Y. Geer, M. Gwadz, D. He, D.I. Hurwitz, J.D. Jackson, Z. Ke, C.J. Lanczycki, C.A. Liebert, C. Liu, F. Lu, G.H. Marchler, M. Mullokandov, B.A. Shoemaker, V. Simonyan, J.S. Song, P.A. Thiessen, R.A. Yamashita, J.J. Yin, D. Zhang and S.H. Bryant, 2005. CDD: A Conserved Domain Database for protein classification. Nucleic Acids Res., 33: D192-196.
65. Dey, S., D. Dou, L.S. Tisa and B.P. Rosen, 1994. Interaction of the catalytic and the membrane subunits of an oxyanion-translocating ATPase. Arch. Biochem. Biophys., 311: 418-24.

66. Chen, C.M., T.K. Misra, S. Silver and B.P. Rosen, 1986. Nucleotide sequence of the structural genes for an anion pump. The plasmid-encoded arsenical resistance operon. J. Biol. Chem., 261: 1503015038.

67. Li, J. and B.P. Rosen, 2000. The linker peptide of the ArsA ATPase. Mol. Microbiol., 35: 361-367.

68. Lanzilotta, W.N., K. Fisher and L.C. Seefeldt, 1997. Evidence for electron-transfer dependent formation of a nitrogenase iron proteinmolybdenum-iron protein tight complex. The role of aspartate 39. J. Biol. Chem., 272: 4157-4165.

69. Kaur, P. and B.P. Rosen, 1993. Complementation between nucleotide binding domains in an aniontranslocating ATPase. J. Bacteriol., 175: 351-357.

70. Ching, M.H., P. Kaur, C.E. Karkaria, R.F. Steiner and B.P. Rosen, 1991. Substrate-induced dimerization of the ArsA protein, the catalytic component of an anion-translocating ATPase. J. Biol. Chem., 266: 2327-2332.

71. Bhattacharjee, H., J. Li, M.Y. Ksenzenko and B.P. Rosen, 1995. Role of cysteinyl residues in metalloactivation of the oxyanion-translocating ArsA ATPase. J. Biol. Chem., 270: 11245-11250.

72. Lahiri, S., L. Pulakat and N. Gavini, 2008. Functional participation of a nifH-arsA2 chimeric fusion gene in arsenic reduction by Escherichia coli. Biochem. Biophys. Res. Commun., 368: 311317. 